\title{
Impact of clinical severity on outcomes of mentalisation-based treatment for borderline personality disorder ${ }^{\dagger}$
}

\author{
Anthony Bateman and Peter Fonagy
}

\section{Background}

Evidence of remission from borderline personality disorder (BPD) without specialised treatment is accumulating.

\section{Aims}

To establish whether specialised treatments are indicated for patients with clinically severe disorder.

\section{Method}

The impact of clinical severity on outcomes of a randomised controlled trial of mentalisation-based treatment (MBT) was contrasted with structured clinical management (SCM). Severity indicators were defined as severity of comorbid psychiatric syndromes, severity of BPD, severity of personality disturbance and severity of symptom distress. Logistic regressions were used to predict the likelihood of recovery at 18 months, and mixed-effects regression analysis was applied to examine the association of severity and rates of improvement across time in the two treatment groups.

\section{Results}

None of the severity criteria predicted outcome at the end of treatment on logistic regression. However, testing the significance of distribution of cases of recovery $v$. nonrecovery suggested that multiple Axis II diagnoses and symptom distress influenced outcomes.

\section{Conclusions}

Borderline personality disorder with significant Axis ॥ comorbidity is a possible but uncertain indicator for specialist treatment. Patients whose only personality disorder diagnosis is BPD do equally well with SCM. Prospective studies are needed.

\section{Declaration of interest}

None.
Borderline personality disorder (BPD) is a complex mental disorder of variable severity. Randomised controlled trials (RCTs) have shown a number of specialist psychosocial treatments to be more effective than treatment as usual, ${ }^{1-5}$ but not necessarily more effective than well-organised general psychiatric treatment. ${ }^{6-8}$ However, this evidence base does not guide clinicians as to who is best referred for the more specialised treatment and who would do equally well with non-specialist intervention. In addition, there are limitations to the data; key clinical groups are often excluded, for example men or individuals with certain comorbid personality disorders such as antisocial type disorder. Additional data from observational studies indicate high rates of remission in BPD in less severely affected patients, ${ }^{9,10}$ that is in those with absence of childhood sexual abuse, no family history of substance use disorder, good vocational record, absence of an anxious cluster personality disorder, low neuroticism and high agreeableness. ${ }^{11}$

On this basis we tested the hypothesis that clinical severity indicates a need for specialist treatment of BPD. ${ }^{12}$ The indicators of severity used in this study were those identified in the exhaustive review of the literature by Crawford et al. ${ }^{13}$ On the basis of earlier reports these authors summarised four main indicators of increasing severity of BPD: level of symptom distress, ${ }^{14}$ number of descriptive criteria met for the disorder, ${ }^{15}$ extent of comorbidity with Axis I disorders, ${ }^{16}$ and comorbidity with other personality disorders, especially in different disorder clusters. ${ }^{17,18}$ Using the data from a recent RCT of mentalisationbased treatment (MBT) compared with structured clinical management (SCM) which had few exclusion criteria - only excluding patients with psychotic disorder, opiate physiological dependence requiring specialist treatment, mental impairment

'See editorial, pp. 163-164, this issue. or evidence of organic brain disorder - it was predicted that higher levels of severity at baseline would increasingly favour MBT over SCM.

\section{Method}

In a recent RCT we compared MBT with manualised SCM in a pragmatic randomised superiority trial involving patients with BPD referred to a north London specialised personality disorder service following a recent crisis episode (attempted suicide or self-harm). The trial was registered with the International Standard Randomised Controlled Trial Number Register (ISRCTN27660668) and approved by the local research ethics committee for Barnet, Enfield and Haringey Mental Health National Health Service (NHS) Trust; full details can be found in the original paper. $^{2}$ Mentalisation-based treatment is a specialised therapy developed to address a hypothetical deficit in the capacity to represent and regulate mental states in patients with BPD. ${ }^{19}$ Structured clinical management is a manualised generic intervention based on routine psychiatric practice but matching the non-specialised features of MBT in terms of intensity, organisation and pharmacological treatment. ${ }^{20}$ Patients assigned to SCM improved on most measures. Patients were treated for 1.5 years with measurement points at baseline and at 6 months, 12 months and 18 months after randomisation. The primary outcome measure was absence of a crisis episode (suicide, self-harm or hospital admission to prevent these) in the previous 6 months. Secondary outcomes were independently rated Global Assessment of Functioning (GAF) scores at the beginning and end of treatment, ${ }^{21}$ and self-reported psychiatric symptoms, social adjustment and interpersonal function, using respectively the Beck 
Depression Inventory (BDI), the Social Adjustment Scale SelfReport (SAS) and the Inventory of Interpersonal Problems (IIP). ${ }^{22-24}$ Medication use was assessed at baseline and at 6-monthly intervals until the end of treatment. Assessors and participants were both masked to assignment. Therapists were randomly assigned to treatment conditions and matched for experience and professional training.

\section{Sample}

The sample comprised 134 participants with BPD from the earlier study assessed using the Structured Clinical Interviews for DSM-IV Axis I and Axis II Disorders (SCID-I and SCID-II) ${ }^{25,26}$ who were randomised to receive MBT $(n=71)$ or SCM $(n=63)$. Inclusion criteria were a diagnosis of BPD; suicide attempt or episode of life-threatening self-harm within the previous 6 months, defined as requiring hospital admission for medical treatment; and age 18-65 years. Exclusion criteria were minimal, with patients with antisocial personality disorder and substance misuse included. Pre-treatment variables and diagnostic data are summarised in the original report. ${ }^{2}$ There was no pre-treatment difference between the groups other than reported rape, which was more common in the MBT group.

\section{Indicators of severity}

Severity was measured at baseline in four ways:

(a) severity of comorbid psychiatric syndromes (number of Axis I diagnoses);

(b) severity of BPD (number of positive criteria met);

(c) severity of personality disturbance (number of comorbid Axis II diagnoses);

(d) severity of symptom distress, indicated by the 90-item Symptom Checklist - Revised (SCL-90-R) Global Severity Index (GSI) scores. ${ }^{27}$

The median was used as a cut-off point for all severity variables - three or more Axis I diagnoses, two or more Axis II diagnoses excluding BPD, a score above 2 on the GSI and six or more positive BPD criteria. All severity variables, except number of BPD criteria met, were also used assuming additivity and continuity. Unexpectedly, we found little redundancy between these indicators as only two of them correlated significantly: the number of Axis I and Axis II diagnoses (Spearman $\rho=0.18$, $P<0.05)$, and Axis I diagnoses and BPD criteria $(\rho=0.21$, $P<0.02)$.

Sample characteristics broken down by the four severity criteria are provided in the online data supplement along with the significant associations. Associations between demographic and clinical features and severity indicators were calculated using appropriate non-parametric statistics including Spearman rho correlations and Wilcoxon rank sum tests (not shown but available from the authors). Notably, unemployment was associated with BPD severity $(\rho=-0.24, P<0.005)$, and gender with number of Axis II diagnoses $(\rho=-0.31, P<0.001)$. These associations were controlled for in all subsequent analyses. Selfrated measures (SCL-90-R, BDI, IIP and SAS) were strongly intercorrelated $(\rho=0.35-0.60)$.

\section{Statistical analysis}

All analyses were carried out using Stata Statistical Software release 12 for Windows. Data analysis was by intention to treat but less than $10 \%$ of data were missing. Logistic regressions were used to predict the likelihood of recovery at 18 months and mixed-effects regressions were applied to examine patterns of change across time on the primary outcome (absence of crisis episode in previous 6-month period) and a selective subset of secondary outcomes. Only primary model parameters directly relevant to the study objectives are presented here. To examine the rate of change we used mixed-effects regression models with participants as random effects. ${ }^{28}$ For the mixed-effect models looking at the pattern of change, the main effect of severity is reported, along with the interaction with treatment group and the linear rate of change for all severity groups from baseline to 18 months for both treatment groups combined. The critical coefficient for each severity parameter was the severity $\times$ group $\times$ time interaction, indicating differential rate of change for the MBT and SCM groups at the severity indicator; a significant coefficient for the three-way interaction indicates that the rate of improvement or deterioration in the MBT group was either slower or faster than in the SCM group at a higher or lower level of severity. As the primary outcome was a composite variable, we followed up significant effects on clinical outcome measures of self-harm, suicidality and hospital admission separately. None of these was normally distributed and hospital admission and suicide were infrequent. Logarithmic transformation was used for self-harm. For suicide attempts and hospital admission we used mixed-effects Poisson regression to create models for count responses. Secondary outcomes were analysed with mixed-effects linear growth curve models for normally distributed variables.

End-of-treatment differences and change over time were analysed using the LOGIT, XTMELOGIT, XTMEPOISSON and XTMIXED procedures in Stata version 12. The four time points were coded as $-3,-2,-1$ and 0 in all models where 6-monthly data were available, thereby implying that regression coefficients involving time measured the linear rate of change from baseline to 18 months and that regression intercepts referenced group differences at the last follow-up point. A likelihood ratio test confirmed that a linear random intercept model best fitted the outcome measures.

Effects for all outcome measures were adjusted by additionally incorporating into all fitted models covariates for rape, because the SCM group was statistically significantly less likely to have this experience, and gender, where this varied with levels of severity. All model parameters for continuous outcome measures are presented as partial standardised effects. To take account of simultaneously testing four independent variables and to protect against type 1 error, we used an alpha level of 0.01 to indicate statistical significance. Complete tables of all modelling results are available from the authors on request.

\section{Results}

Our previous report provided information concerning treatment group differences on both primary and secondary outcome measures. $^{2}$ Only associations with severity indicators are considered here. As was originally reported in both groups, the multilevel mixed-effects logistic regression revealed that the odds ratio (OR) associated with recovery on the primary outcome (no suicide attempt, self-harm or hospital admission in the previous 6 months) increased with time (main effect of time: $\mathrm{OR}=2.98,95 \%$ CI $\left.1.58-5.59, t_{(134)}=3.39, P<0.001, d=0.59\right)$, but the increase was greater in the group randomised to MBT (time $\times$ group interaction $\mathrm{OR}=5.37,95 \%$ CI $1.96-14.69$, $\left.t_{(134)}=3.27, P<0.001, d=0.56\right)$. The number and percentage of recovered participants in the SCM and MBT arms at each observation point are presented in Table 1 for participants grouped according to four criteria for the severity of initial 
Table 1 Proportion of patients recovered at three time points in the two arms of the trial grouped according to four indicators of severity of presentation at baseline

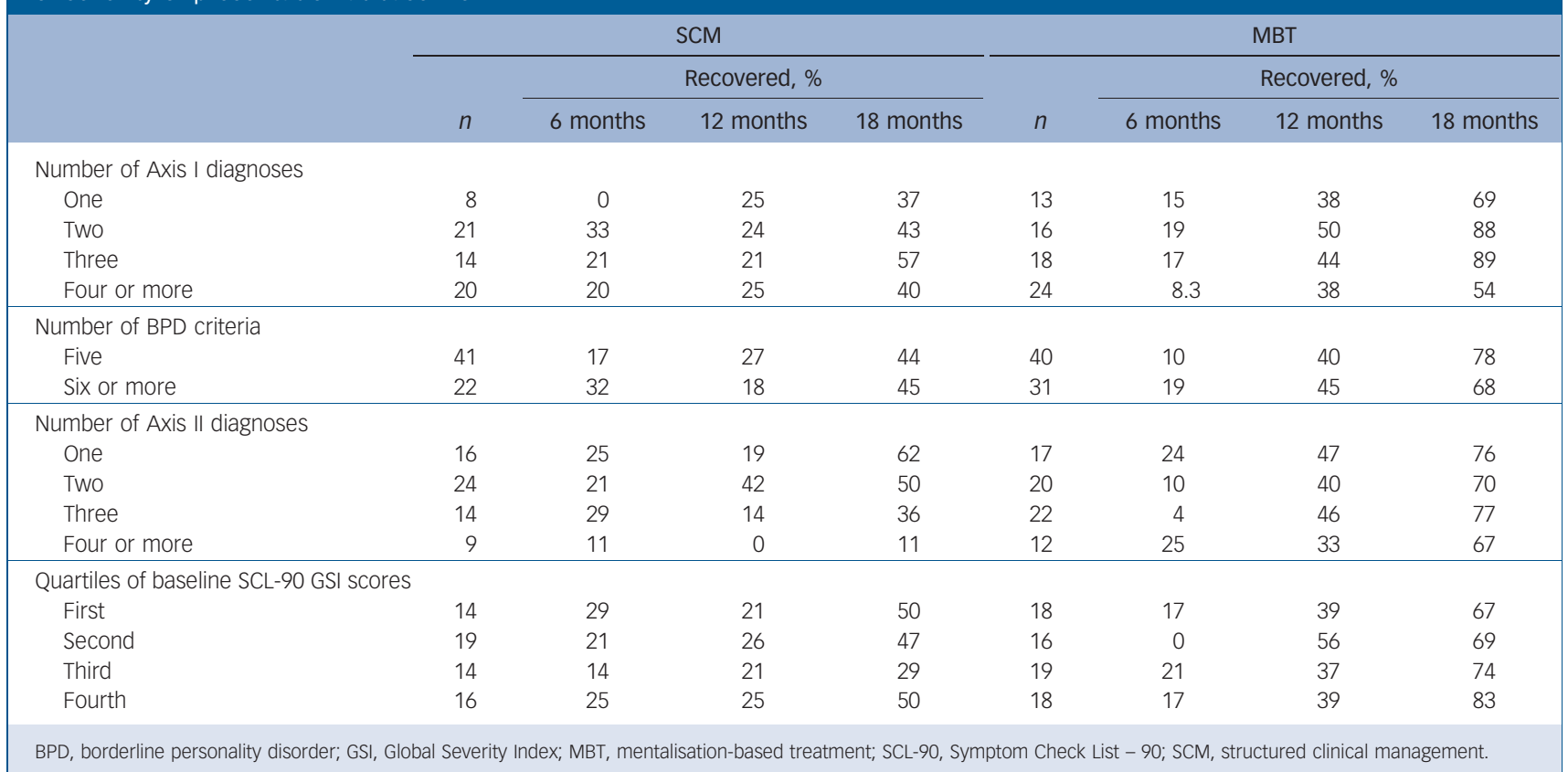

presentation. Table 2 lists the percentage of patients recovered at the end of treatment according to each severity criterion and parameters obtained from mixed-effects regression models for the four binary-coded severity indicators tested.

None of the severity criteria predicted outcome at the end of treatment on logistic regression as a main effect or interaction in combination with condition using a conservative value of $\alpha=0.01$. However, Pearson chi-squared testing of the significance of distribution of recovery and non-recovery in the severely affected group yielded two significant group differences. Those above the median for severity on GSI and number of Axis II variables were more likely to recover in the MBT group than similar patients in the SCM group. Only about a quarter (6 of 23) of patients with three or more Axis II diagnoses recovered at 18 months in SCM, compared with nearly three-quarters (25 of 34 ) of the MBT group $\left(\chi^{2}(1)=12.45, P<0.001\right) ; 78 \%$ of the MBT group with GSI scores above the median for the sample recovered, compared with $40 \%$ of the SCM group $\left(\chi^{2}(1)=10.28, P<0.01\right)$ (Table 2). Table 2 also shows analyses by presence or absence of comorbid cluster A, B or C diagnoses. Only additional diagnosis of cluster $\mathrm{C}$ was significantly associated with increased likelihood of recovery in the MBT group. The number of Axis I diagnoses did not predict the likelihood of recovery overall or selectively in either of the treatment groups. The number of BPD criteria met also failed to correlate with recovery $\left(\rho_{(n=130)}=-0.05\right.$, NS).

Table 2 also contains information concerning the rate of increase in the number of patients who recovered by these criteria. Only the number of Axis II diagnoses yielded significant main or interaction effects. The significant time $\times$ Axis II severity interaction $\left(\beta=-0.011,95 \%\right.$ CI -0.19 to $-0.04, t_{(134)}=-2.87$, $P<0.004, d=0.48)$ suggests that patients with more Axis II diagnoses recovered at a slower rate. Overall, $38 \%$ of those with only one diagnosis in addition to BPD had recovered by 12 months and $64 \%$ by 18 months; only $28 \%$ and $54 \%$ of those with two or more additional diagnoses had recovered at these times. Importantly, the prediction from the baseline number of Axis II diagnoses was significantly stronger for the SCM group, as indicated by the significant three-way interaction (severity effect on group differential rate of change: $\beta=0.11,95 \%$ CI $0.01-0.22, t_{(134)}=2.41, P<0.01$, $d=0.45$ ). To illustrate this interaction, Fig. 1 shows the linear prediction of recovery for each of the treatment groups at linear portions of the time variable at one, two, three or four comorbid Axis II diagnoses. Although the model predicted almost identical rates of recovery for the cases with only BPD diagnosis, the prediction of the rate of recovery decreased with each additional Axis II diagnosis for the SCM group, whereas it remained essentially unaltered for the MBT group. No similar association of severity indicators with recovery rates was evident for Axis I, symptom distress or BPD severity criteria.

We explored whether the additional benefit from MBT affected the three indicators contributing to the definition of recovery: hospital admission, self-harm or suicide attempt. We used mixed-effects Poisson regressions to model the frequency data associated with hospital admission and suicide attempt. The Poisson regression for the number of suicide attempts yielded no significant interaction with the number of Axis II diagnoses: incidence rate ratio (IRR) $1.04,95 \%$ CI $0.84-1.29, t_{(134)}=0.4$, NS, $d=0.07$. The interaction between treatment group and time also missed the Bonferroni-adjusted significance (IRR $=0.56$, $95 \%$ CI $\left.0.31-0.99, t_{(134)}=1.96, P<0.05, d=0.34\right)$. Similar negative results emerged in relation to number of hospital admissions. The only component of recovery to reflect the impact of Axis II diagnosis was self-harm. The Poisson regression yielded a main effect for severity $\left(\mathrm{IRR}=1.98,95 \%\right.$ CI $1.21-3.22, t_{(134)}=2.76$, $P<0.006, d=0.48)$ and interaction with time (IRR $=1.27,95 \%$ CI $\left.0.47-1.44, t_{(134)}=3.81, P<0.0001, d=0.66\right)$. A significant three-way interaction indicated that the reduction of self-harm over the course of treatment was substantial only for those with one or two Axis II diagnoses in the SCM group, whereas the moderating effect was not apparent in the MBT group $\left(\mathrm{IRR}=0.79,95 \%\right.$ CI $\left.0.66-0.93, t_{(134)}=2.75, P<0.006, d=0.48\right)$. Mixed-effects regression using logarithms of self-harm events yielded a similar three-way interaction and effect size $(\beta=-0.11$, $95 \%$ CI -0.24 to $\left.-0.03, t_{(134)}=-2.56, P<0.01, d=0.44\right)$. Overall, MBT was more effective than SCM for individuals with a higher number of Axis II diagnoses in reducing self-harm.

We restricted analysis of secondary outcomes to the severity indicator that yielded significant interactions with the primary 



Fig. 1 Predicted recovery according to Axis II pathology. (a) One Axis II diagnosis; (b) two Axis II diagnoses; (c) three Axis II diagnoses; (d) four Axis II diagnoses. MBT, mentalisation-based treatment; SCM, structured clinical management.

outcome measure. Three secondary outcomes were considered, namely scores on the BDI, IIP and SAS. Linear regression predicting end-of-treatment effects and mixed-model random effects regressions are summarised in Table 3. For two of the three variables there was a three-way, time $\times$ number of Axis II diagnoses $\times$ group interaction, with self-rated depression scores declining and interpersonal function improving faster in the MBT arm for individuals with three or four diagnoses. For BDI scores the contrast between SCM and MBT groups was not significant at 12 months and 18 months in patients with one or two diagnoses $(\beta=-1.09,95 \% \mathrm{CI}-4.21$ to $2.12, z=-0.68$, NS; $\beta=-1.91,95 \%$ CI -5.43 to $1.60, z=1.07$, NS, respectively). In contrast, BDI scores were marginally significantly lower for individuals with three or more diagnoses in the MBT treatment arm at 12 months $(\beta=-4.42,95 \%$ CI -8.07 to -0.78 , $z=-2.38, P<0.017)$ and this difference increased by 18 months $(\beta=-6.94,95 \%$ CI -10.9 to $-2.88, z=-3.35, P<0.001)$.

The IIP scores were not significantly lower at 18 months in the MBT group even for individuals with only a BPD diagnosis and no comorbidity $(\beta=-0.28,95 \%$ CI -0.54 to $-0.01, z=-2.01$, $P<0.04)$. The size of the difference was marked at 18 months for individuals with two or three Axis II diagnoses $(\beta=-0.372$, $95 \% \mathrm{CI}-0.55$ to $-0.12, z=-4.20, \quad P<0.0001 ; \beta=-0.47$, $95 \%$ CI -0.67 to $-0.27, z=-4.7, P<0.0001$, respectively) and became even larger for those with four diagnoses $(\beta=-0.576$, $95 \%$ CI -0.90 to $-0.25, z=3.49, P<0.0001)$. There was no significant interaction associated with social adjustment scores, but the MBT group showed better function independently of the number of Axis II diagnoses.

To test whether baseline correlates of the number of Axis II diagnoses (such as social isolation, unemployment, education level, early loss, sexual abuse, physical abuse, history of antisocial behaviour and GAF score) or better medical management of the multiple personality disorder diagnosis group could account for the greater benefit from MBT, we examined the correlation between number of personality disorder diagnoses and these risk variables, but identified no significant association except gender and baseline GAF score $\left(\rho_{(134)}=-0.24, P<0.006\right)$. Including GAF in addition to gender in the models did not alter any of the findings. The use of medication, number of different classes of medication used or use of antidepressants, mood stabilisers or benzodiazepines were also not associated with group $\times$ time interactions. There was some indication of a relatively more rapid reduction in antipsychotic use in patients with a greater number of Axis II diagnoses (OR $=-1.57,95 \%$ CI -3.32 to 0.18 , $z=-1.76, \quad P<0.08)$ but this did not change any of the associations reported.

\section{Discussion}

The results suggest that increasing severity of BPD, based on Axis II comorbidity and symptom severity, may predict greater benefit from MBT over SCM, but the evidence is limited. None of the severity criteria predicted outcome at the end of treatment on logistic regression as a main effect or interaction in combination with condition using Bonferroni correction. Complex or severe personality disorder, where criteria for more than one such disorder are met, may entail a range of correlated factors such as greater likelihood of being unemployed and greater social and interpersonal dysfunction, ${ }^{29,14}$ accounting for the relatively worse outcomes overall. The variable prediction from severity indicators 

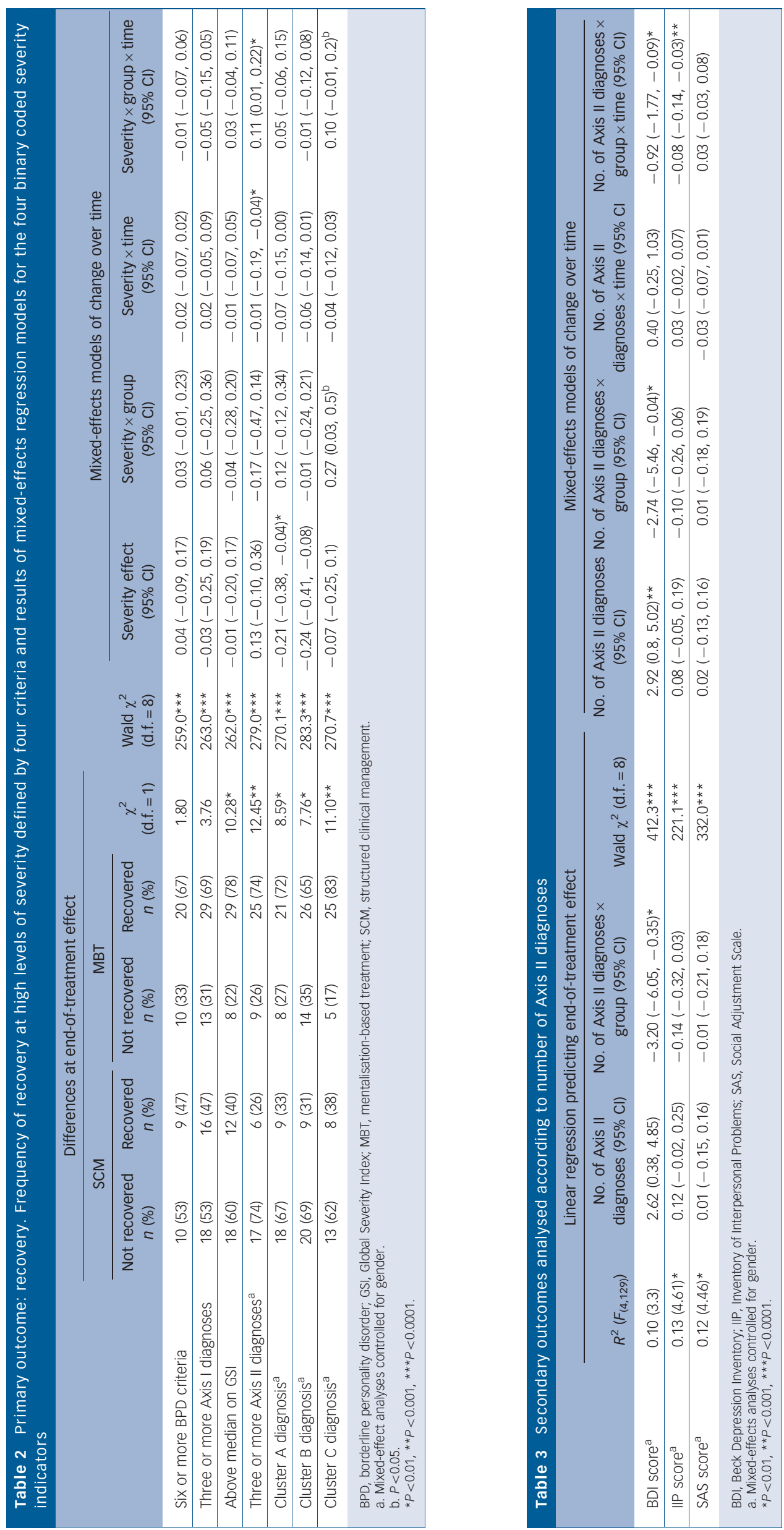
on outcomes of treatment contrasts with findings from some larger, longer-term follow-along studies. ${ }^{30-32}$ Gunderson et al in a prospective longitudinal study found that severe baseline psychopathology (suggested by higher levels of BPD criteria and functional disability) along with childhood trauma predicted poor outcomes over 2 years. $^{30}$ Other studies, mostly using retrospectively collected data, have identified other predictors of variations in the longitudinal course of the disorder, including affective disorder and other Axis I disorders, substance use, dysphoria and comorbid personality disorders. Only the last factor might have influenced treatment outcomes in our smaller sample, in keeping with the finding in a prospective follow-along study that absence of comorbid cluster C improved likelihood of remission over time. ${ }^{33}$

The results tentatively suggest that the complexity of the personality disorder, suggested by the moderating effect on treatment of additional Axis II diagnoses, has a negative impact on treatment outcomes. In a study on the long-term course of global functioning in patients in therapy with mixed personality disorder, Kvarstein \& Karterud also found that comorbid avoidant traits, an indicator of increasing complexity, were a negative prognostic factor. ${ }^{34}$ Long-term functional outcomes, including social impairment, are problematic in $\mathrm{BPD} .{ }^{8}$ It is possible that the combination of BPD and avoidant features characteristic of other clusters of personality disorder may increase difficulty for treatment because of the greater social impairment, ${ }^{35}$ which maintains impairment of emotion regulation by limiting exposure to potentially corrective social influences. ${ }^{36}$

\section{Components of therapy}

If it is confirmed that MBT is more beneficial for patients whose BPD is embedded in other personality problems, how can we explain the paradox that treatment specifically designed for BPD may be beneficial for those with additional problems beyond the target of the therapy? We have limited data to answer this question. We may conjecture, first, that although MBT was designed for BPD it may have broader scope. Mentalising is a key component of self-identity and a central aspect of interpersonal relationships and social function; thus a focus on mentalising might have an impact on a range of disordered mental processes whatever the pathology. If personality disorder is conceptualised as a serious impairment in interpersonal relationships, intimacy, identity and self- direction, ${ }^{37}$ enhancing mentalising might benefit the disorder as a whole regardless of subtype. In contrast, SCM is more restricted in scope, which makes it less relevant to patients' functional problems.

Second, personality disorders as discrete diagnostic groupings each challenge therapy with specific treatment-interfering behaviours. Various treatment models for BPD may act through a dual set of mechanisms: a set of modality-specific mechanisms, designed to maintain engagement in treatment and address aspects of the patient's presentation that potentially undermine a second generic rehabilitative component that is, perhaps, embedded in the therapeutic relationship. We might argue that SCM contains many elements of such a rehabilitative therapeutic process, but the mentalisation focus in MBT ensures that interpersonal challenges linked to multiple personality disorder diagnoses are dealt with, allowing the generic therapeutic component shared with SCM to be delivered.

Third, the possible, albeit limited, advantage of MBT might be due to its reduction of the potential for psychotherapy to harm patients with BPD. $^{38}$ Therapists delivering SCM may have inadvertently caused some harm. Betan et al found that therapist countertransference to patients with cluster C personality disorders was parental/protective, with therapists wishing to protect and nurture the patient above and beyond normal positive feelings towards the patient. ${ }^{39}$ Mentalisation-based treatment specifically cautions against overprotective/nurturing interactions because of the danger of overstimulation of the attachment system. $^{40}$

\section{Limitations of the study}

There are a number of limitations to this report. First, the trial was not powered to examine moderating variables. Specially designed investigations or a full meta-analytical study are needed. The results must be considered as tentative observations providing grounds for future systematic enquiry and are not robust enough to guide clinical decision-making. Second, multiple parameters of severity were used because of lack of agreement in the literature on definition, but we attempted to control for the increased risk of type 1 error by using conservative alpha values. Third, the sample may not be representative of people with BPD, although exclusion criteria were kept to a minimum and this was a pragmatic trial embedded in clinical services. Fourth, a specific measure of overall social and personal adaptation was not available. Finally, there was no measure of mentalisation to allow assessment of the purported process of change leading to the possible improved outcomes of patients with more complex personality disorder treated with MBT.

\section{Implications}

Overall, the results tentatively suggest that greater complexity indicates the need for specialist treatment. However, the observations remain preliminary. If they are confirmed by further studies, this would allow appropriate selection at assessment of patients for specialist treatment and the development of an evidence-based stepped-care approach. Given that we found no correlation between measures of severity, we need to be cautious about what current measures indicate. A new continuous measure of personality disorder severity, as suggested in current proposals for a new classificatory system in ICD $-11,{ }^{41}$ may be necessary. It would be valuable, in addition, to have indicators of improvement in therapy during the course of treatment by monitoring progress to facilitate an increasingly informed stepped-care approach.

Anthony Bateman, FRCPsych, Halliwick Personality Disorder Service and Anna Freud Centre, London; Peter Fonagy, PhD, Psychoanalysis Unit, University College London, UK

Correspondence: Professor Anthony Bateman, Halliwick Personality Disorder Service, St Ann's Hospital, St Ann's Road, London N15 3TH, UK. Email: anthony@mullins.plus.com

First received 17 Sep 2012, final revision 7 Apr 2013, accepted 17 Apr 2013

\section{Funding}

The original trial was funded by the Borderline Personality Disorder Research Foundation.

\section{References}

1 Linehan MM, Comtois KA, Murray AM, Brown MZ, Gallop RJ, Heard HL, et al. Two-year randomized controlled trial and follow-up of dialectical behavior therapy vs therapy by experts for suicidal behaviors and borderline personality disorder. Arch Gen Psychiatry 2006; 63: 757-66.

2 Bateman A, Fonagy P. Randomized controlled trial of outpatient mentalization-based treatment versus structured clinical management for borderline personality disorder. Am J Psychiatry 2009; 166: 1355-64.

3 Gieson-Bloo J, van Dyck R, Spinhoven P, van Tilburg W, Dirksen C, van Asselt $T$, et al. Outpatient psychotherapy for borderline personality disorder; randomized trial of schema-focused therapy vs transference focused therapy. Arch Gen Psychiatry 2006; 63: 649-58. 
4 Davidson K, Norrie J, Tyrer P, Gumley A, Tata P, Murray H, et al. The effectiveness of cognitive behavior therapy for borderline personality disorder: results from the borderline personality disorder study of cognitive therapy (BOSCOT) trial. J Pers Disord 2006; 20: 450-65

5 Clarkin JF, Levy KN, Lenzenweger MF, Kernberg O. Evaluating three treatments for borderline personality disorder. Am J Psychiatry 2007; 164: 922-8.

6 Chanen AM, Jackson HJ, McCutcheon LK, Jovev M, Dudgeon P, Yuen HP, et al. Early intervention for adolescents with borderline personality disorder using cognitive analytic therapy: randomised controlled trial. Br J Psychiatry 2008; 193: 477-84.

7 McMain S, Guimond T, Cardish R, Streiner D, Links P. Clinical outcomes and functioning post-treatment: a two-year follow-up of dialectical behavio therapy versus general psychiatric management for borderline personality disorder. Am J Psychiatry 2012; 169: 650-61.

8 Bateman A. Treating borderline personality disorder in clinical practice. Am J Psychiatry 2012; 169: 1-4.

9 Zanarini MC, Frankenburg FR, Hennen J, Reich DB, Silk KR. The McLean Study of Adult Development (MSAD): overview and implications of the first six years of prospective follow-up. J Pers Disord 2005; 19: 505-23.

10 Skodol AE, Gunderson J, Shea MT, McGlashan T, Morey LC, Sanislow C, et al. The Collaborative Longitudinal Personality Disorders Study (CLPS): overview and implications. J Pers Disord 2006; 19: 487-504.

11 Zanarini MC, Frankenburg FR, Reich DB, Fitzmaurice G. The 10-year course of psychosocial functioning among patients with borderline personality disorder and Axis II comparison subjects. Acta Psychiatr Scand 2010; 122: 103-9.

12 Oldham J, Skodol AE, Bender DS (eds). Textbook of Personality Disorders. American Psychiatric Publishing, 2005.

13 Crawford MJ, Koldobsky N, Mulder R, Tyrer P. Classifying personality disorder according to severity. J Pers Disord 2011; 25: 321-30.

14 Gunderson J, Links P, Reich J. Competing models of personality disorders. J Pers Disord 1991; 5: 60-8.

15 Arntz A, van den Hoorn M, Cornelis J, Verheul R, van den Bosch W, de Bie A. Reliability and validity of the borderline personality disorder severity index. J Pers Disord 2003; 17: 45-59.

16 Akiskal HS. Demystifying borderline personality: critique of the concept and unorthodox reflections on its natural kinship with the bipolar spectrum. Acta Psychiatr Scand 2004; 110: 401-7.

17 Tyrer $\mathrm{P}$, Johnson $\mathrm{T}$. Establishing the severity of personality disorder. Am J Psychiatry 1996; 153: 1593-7.

18 Tyrer $\mathrm{P}$, Mulder R. Management of complex and severe personality disorders in community mental health services. Curr Opin Psychiatry 2006; 19: 400-4.

19 Fonagy $\mathrm{P}$. An attachment theory approach to treatment of the difficult patient. Bull Menninger Clin 1998; 62: 147-69.

20 Bateman A, Krawitz R. Borderline Personality Disorder: An Evidence-Based Guide for Generalist Mental Health Professionals. Oxford University Press, 2013.

21 American Psychiatric Association. Diagnostic and Statistical Manual of Mental Disorders (4th edn, Text Revision) (DSM-IV-TR). APA, 2000.

22 Beck A, Ward $\mathrm{CH}$, Mendelson M, Mock J, Erbaugh J. An inventory for measuring depression. Arch Gen Psychiatry 1961; 4: 561-71.

23 Weissman MM, Bothwell S. Assessment of social adjustment by patient self-report. Arch Gen Psychiatry 1976; 33: 1111-5.
24 Horowitz LM, Rosenberg SE, Baer BA, Ureno G, Villasenor G. Inventory of interpersonal problems: psychometric properties and clinical applications. J Consult Clin Psychol 1988; 56: 885-92.

25 First MB, Gibbon M. Williams JBW, Spitzer R. Structured Clinical Interview for DSM-IV Axis I Disorders (SCID-I). Biometrics Research Department, New York Psychiatric Institute, 1995.

26 First MB, Gibbon M, Williams JBW, Spitzer R. Structured Clinical Interview for DSM-IV Axis II Personality Disorders (SCID-II). Biometrics Research Department, New York Psychiatric Institute, 1995.

27 Derogatis LR. SCL-90R: Administration, Scoring and Procedures - Manual II. Clinical Psychometric Research, 1983.

28 Rabe-Hesketh S, Skrondal A. Multilevel and Longitudinal Modeling Using STATA (2nd edn). Stata Press, 2008

29 Tyrer P. Personality disorder and public mental health. Clin Med 2008; 8 423-7.

30 Gunderson JG, Daversa MT, Grilo CM, McGlashan TH, Zanarini MC, Shea MT, et al. Predictors of 2-year outcome for patients with borderline personality disorder. Am J Psychiatry 2006; 163: 822-6.

31 Links P, Heslegrave R, Van Reekum R. Prospective follow-up study of borderline personality disorder: prognosis, prediction of outcome, and Axis II comorbidity. Can J Psychiatry 1998; 43: 265-70.

32 Paris J, Brown R, Nowlis D. Longterm follow-up of borderline patients in a general hospital. Compr Psychiatry 1987; 28: 530-5.

33 Zanarini MC, Frankenburg FR, Hennen J, Reich DB, Silk KR. Prediction of the 10-year course of borderline personality disorder. Am J Psychiatry 2006; 163 : 827-32.

34 Kvarstein E, Karterud S. Large variations of global functioning over five years in treated patients with personality traits and disorders. J Pers Disord 2012; 26: 141-61.

35 Wilberg T, Karterud S, Pederson G, Urnes O. The impact of avoidant personality disorder on psychosocial impairment is substantial. Nord J Psychiatry 2009; 63: 1-7.

36 Shea MT, Stout RL, Gunderson J, Morey LC, Grilo C, McGlashan TH, et al. Short-term diagnostic stability of schizotypal, borderline, avoidant, and obsessive-compulsive personality disorders. Am J Psychiatry 2002; 159: 2036-41.

37 Skodol AE, Clark LA, Bender D, Krueger RF, Morey LC, verheul R, et al. Proposed changes in personality and personality disorder assessment and diagnosis for DSM-5 Part I: Description and rationale. Personal Disord 2011; 2: 4-22.

38 Fonagy $\mathrm{P}$, Bateman A. Progress in the treatment of borderline personality disorder. Br J Psychiatry 2006; 188: 1-3.

39 Betan E, Heim AK, Zittel Conklin C, Westen D. Countertransference phenomena and personality pathology in clinical practice: an empirical investigation. Am J Psychiatry 2005; 162: 890-8.

40 Fonagy $\mathrm{P}$, Bateman $\mathrm{A}$. The development of borderline personality disorde - a mentalizing model. J Pers Disord 2008; 22: 4-21.

41 Tyrer $\mathrm{P}$, Crawford $\mathrm{M}$, Mulder R, Blashfield R, Farnam A, Fossati A, et al. The rationale for the reclassification of personality disorder in the 11 th revision of the International Classification of Diseases (ICD-11). Personal Ment Health 2011; 5: 246-59. 